\title{
Superior stability secured by a four-phase cathode electrolyte interface on Ni-rich cathode for lithium ion batteries
}

Shaodian Yang ${ }^{1,2, \#}$, Qinglu Fan ${ }^{1,2, \#}$, Zhicong Shi ${ }^{1,2 *}$, Liying Liu ${ }^{1,2^{*}}$, Jun Liu ${ }^{1,2}, X_{i} \mathrm{Ke}^{1,2}$, Jianping Liu ${ }^{1,2}$, Chaoyu Hong ${ }^{3}$, Yong Yang ${ }^{3}$ and Zaiping Guo ${ }^{*}$

${ }^{1}$ Smart Energy Research Centre, School of Materials and Energy, Guangdong University of Technology, Guangzhou, 510006, PR China

2 Guangdong Engineering Technology Research Center for New Energy Materials and Devices, Guangzhou, 510006, PR China

${ }^{3}$ Department of Chemistry, Xiamen University, Xiamen, 361005, PR China

${ }^{4}$ Institute for Superconducting \& Electronic Materials, School of Mechanical, Materials and Mechatronics Engineering, University of Wollongong, Wollongong, NSW 2522, Australia

*Corresponding authors: zhicong@gdut.edu.cn, (Z. Shi), liyingliusy@163.com (L. Liu),zguo@uow.edu.au (Z.Guo)

\# Shaodian Yang and Qinglu Fan contributed equally. 
(a)

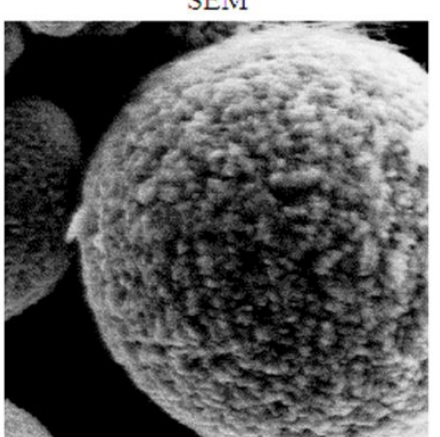

$\mathrm{Mn}$

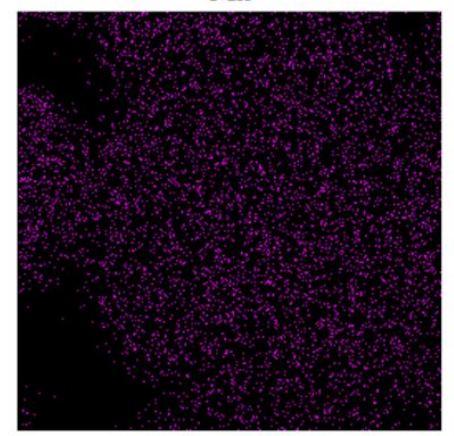

SEM
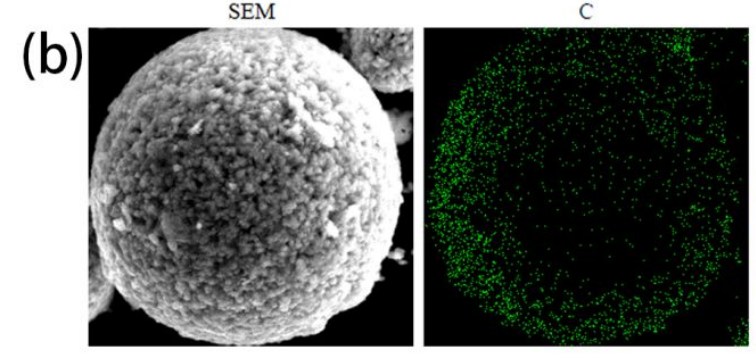

$\mathrm{Mn}$
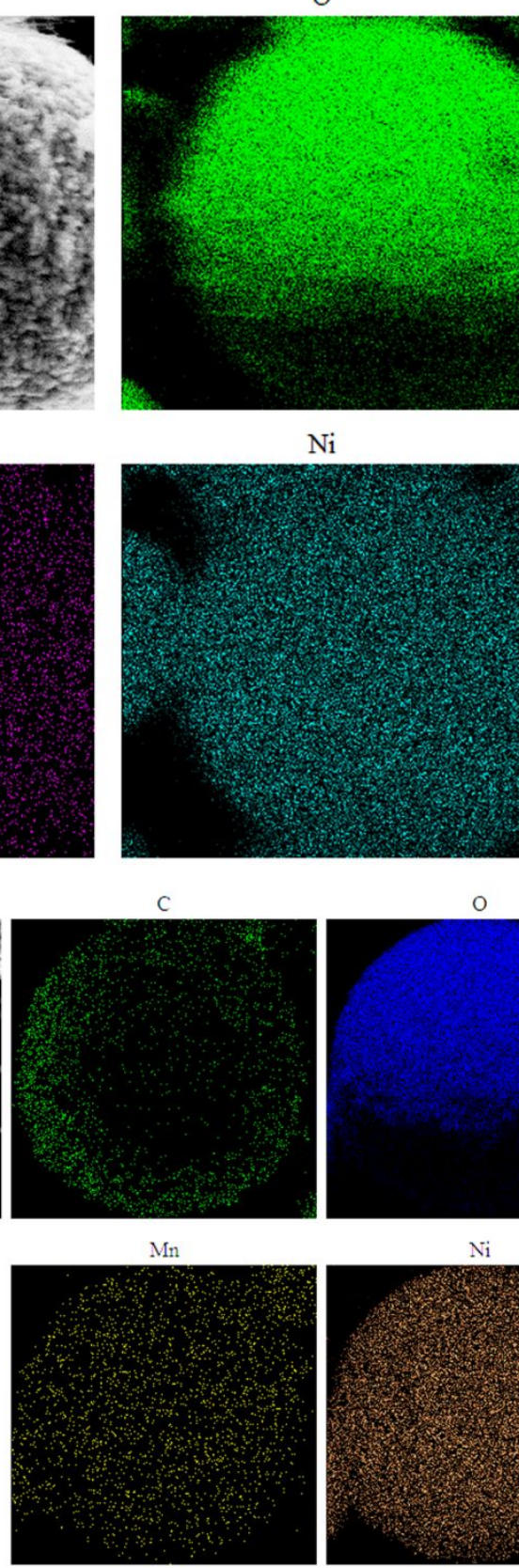

$\mathrm{Ni}$

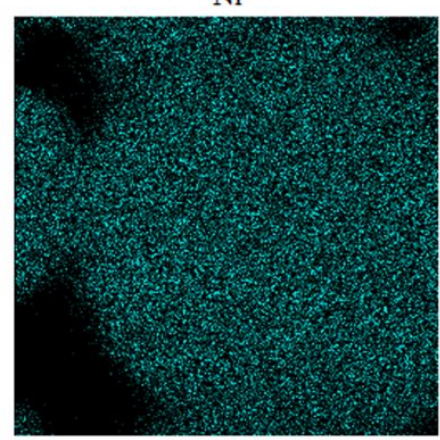

o

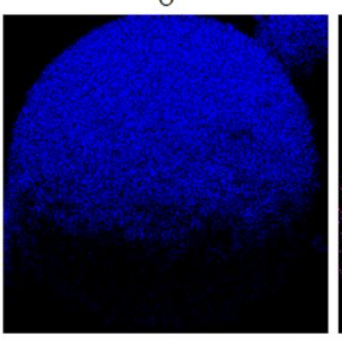

$\mathrm{Ni}$

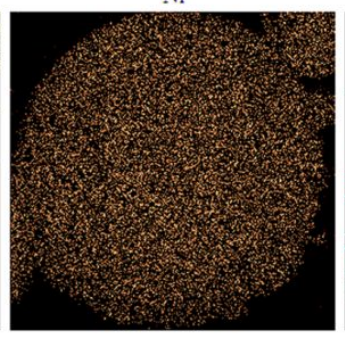

P

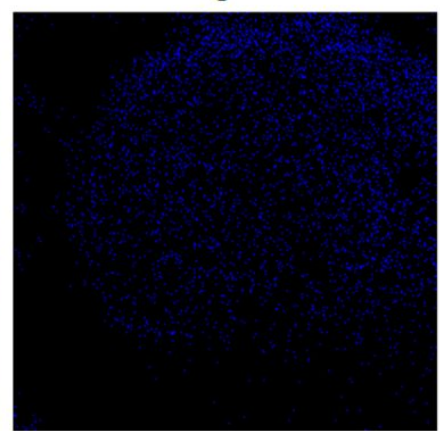

Co

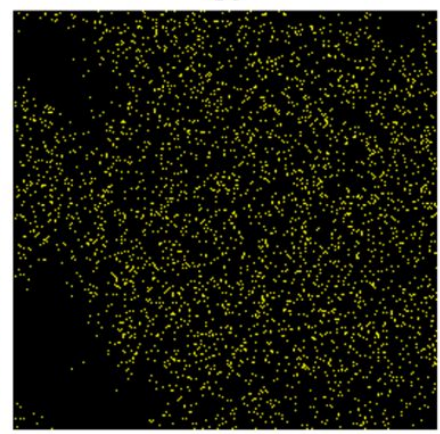

$\mathrm{P}$

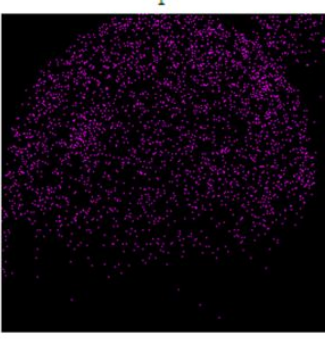

Co

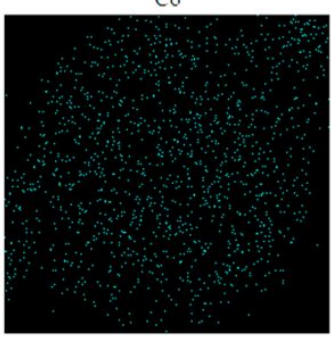

Figure S1 The EDS elemental mappings of C, O, P, Co, Ni, Mn of LPO-NCM (a) and CNT-LPO-NCM (b).

Both $\mathrm{C}$ and $\mathrm{P}$ elements are uniformly covered on the surface of the layered oxide, which indicates that the CNTs and $\mathrm{Li}_{3} \mathrm{PO}_{4}$ are uniformly coated on the surface of the layered oxide to form solid electrolyte interface (Figure S1). 


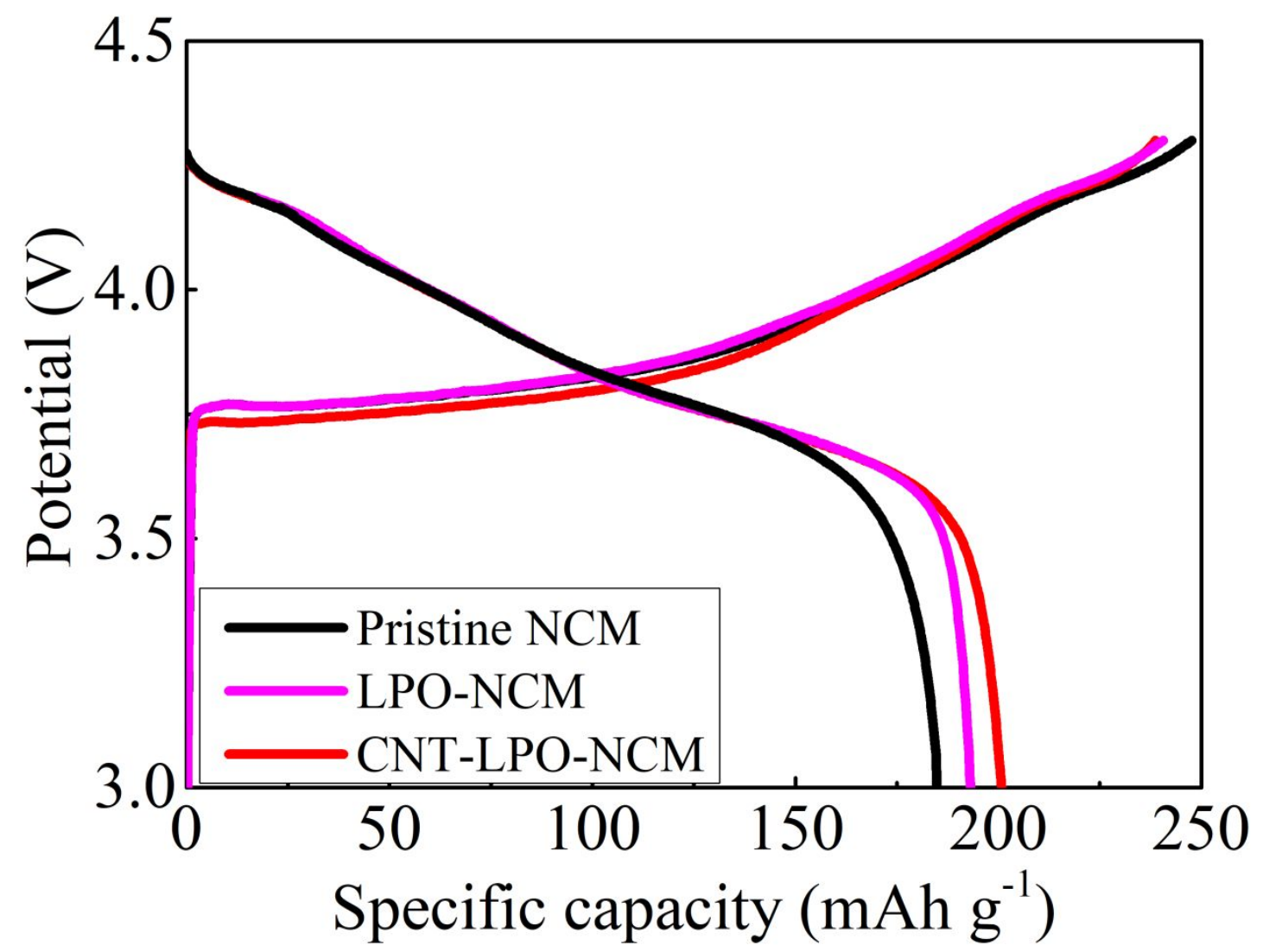

Figure S2 The initial charge and discharge curves of Pristine NCM, LPO-NCM and CNT-LPO-NCM between $3 \mathrm{~V}$ and $4.3 \mathrm{~V}$ at room temperature.

The initial coulomb efficiency of pristine NCM, LPO-NCM and CNT-LPO-NCM are $74.8 \%, 80.7 \%$ and $85.1 \%$, respectively (Figure S2). The initial discharge specific capacities of pristine NCM, LPO-NCM and CNT-LPO-NCM are 184.8, 193.8, and $202.8 \mathrm{~mA} \mathrm{~h} \mathrm{~g}^{-1}$, respectively. 


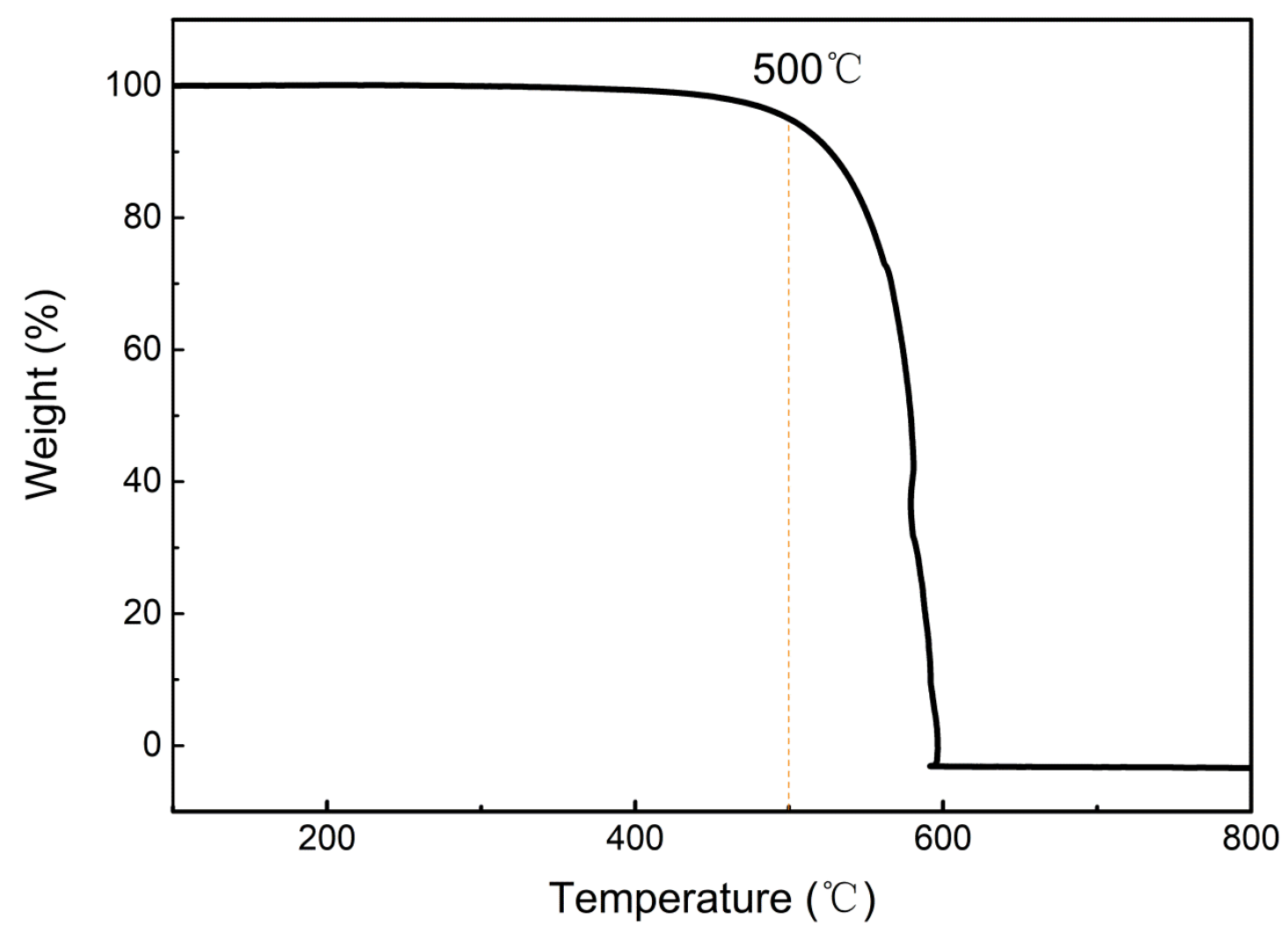

Figure S3 The TGA curves of CNTs in air atmosphere.

When the temperature is increased to $500^{\circ} \mathrm{C}$, the weight of CNTs is only slightly lost in air atmosphere. But the CNTs will burn out in air after the temperature increased to $600^{\circ} \mathrm{C}$. In order to ensure the integrity of the CNTs is maintained in the coating layer, the heat treatment process of modified NCM is carried out in the Ar atmosphere.
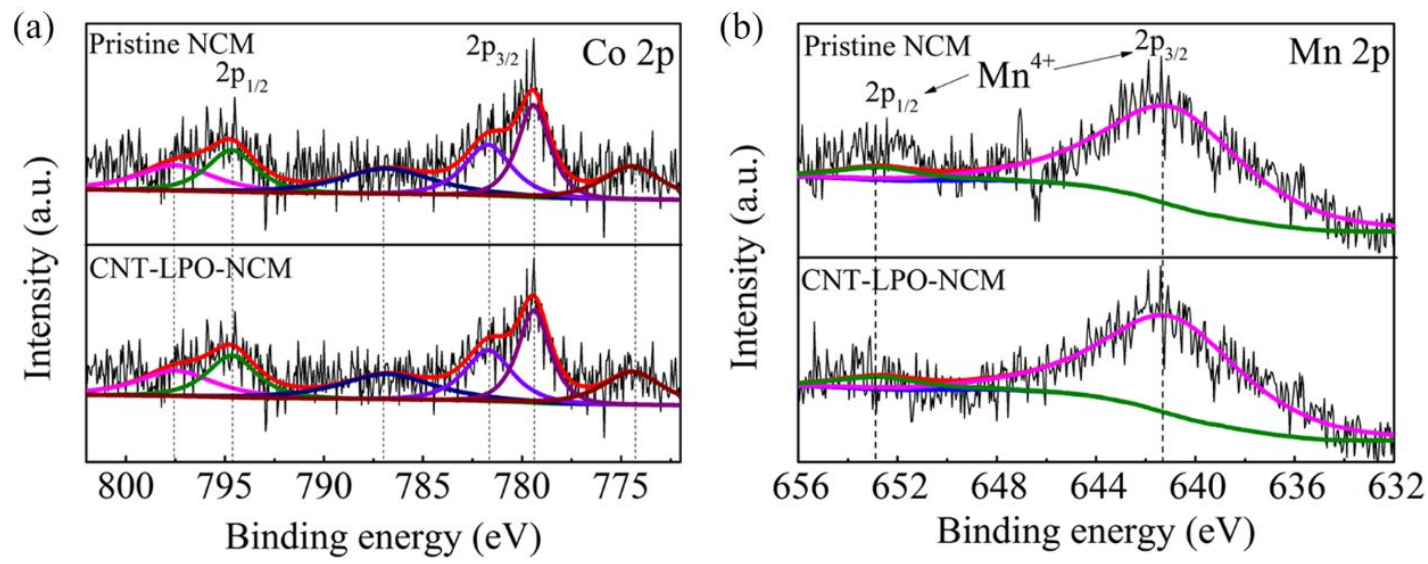

Figure S4 XPS spectra of pristine NCM and CNT-LPO-NCM: (a) Co 2p, (b) Mn 
$2 p$.

Table S1 Rietveld Refinement Results of XRD Data for Pristine NCM, LPO-NCM and CNT-LPO-NCM

\begin{tabular}{cccc}
\hline & Pristine NCM & LPO-NCM & CNT-LPO-NCM \\
\hline $\mathrm{a}(\AA)$ & 2.8684 & 2.8692 & 2.8703 \\
$\mathrm{c}(\AA)$ & 14.1802 & 14.1813 & 14.1915 \\
unite volume $\left(\AA^{3}\right)$ & 101.1 & 101.1 & 101.3 \\
$\mathrm{c} / \mathrm{a}$ & 4.9436 & 4.9425 & 4.9442 \\
\hline
\end{tabular}

